\title{
A FUZZY METRIC FOR ASSESSING THE PRODUCIBILITY OF STRAIGHTENING IN EARLY DESIGN
}

\author{
J-D Caprace, F. Aracil Fernandez, N. Losseau and P. Rigo, University of Liege, Belgium
}

\section{SUMMARY}

Since several years, the large shipyards use more and more thin plates to build up stiffened panels in order to decrease the structural weight. The major problem relating to the utilization of thin plates is the occurrence of welding distortions that have to be eliminated for esthetical and service reasons. This straightening operation involves significant costs and it seems thus important to characterize its economical impact on the global hull fabrication.

This paper presents a way to minimize cost in shipbuilding industry by the implementation of a producibility fuzzy metric in order to obtain a better knowledge of the straightening process. Attention has been focused on the definition of fuzzy rules by experts but also on the optimisation of the decision surface to reduce the error compared to actual measurement points.

\section{INTRODUCTION}

Capturing producibility during the design of a new ship is one of the most difficult parts of the design process. The factors influencing the manufacturing are always changing. It is only once the production plans are finalized that is it possible to make a direct and more reliable cost assessment. However, the pressure to deliver a new vessel on time and within the budget means that construction must begin before such detailed production related aspects are finalized. Construction cost must be tracked continuously during the design process to ensure the project remains viable to both shipyard and customer particularly when late changes have considerable cost impacts. Unfortunately, cost is often a secondary consideration for the designers and the engineers concentrating on delivering the technical aspects of the new design. However, to succeed commercially, shipyards must be able to accurately assess their costs.

This paper presents a way to minimize cost in shipbuilding industry by the implementation of a producibility cost metric in order to obtain a better knowledge of the straightening operations.

\subsection{STRAIGHTENING OPERATIONS}

Since several years, the big shipyards use more and more thin plates to build up the stiffened panels in order to decrease the structural weight of ships [4]. The major problem relating to the utilization of thin plates is the appearance of welding distortions that have to be eliminated (see Figure 1). The straightening is the process that consists to eliminate these distortions in order to reduce the structure flatness for esthetical or service reasons (see Figure 2).

As the straightening process involves non negligible labour costs [8]; it seems interesting to estimate the straightening impact on the production workload in order to improve the research in the following domains: production simulation, cost assessment of ship hull, structure optimisation, design for production, etc.

In shipbuilding research, some models to predict roughly the welding distortions have been developed [3, 7] but very few regarding the straightening cost. Due to the natural uncertainties associated to the relevant cost data and the variability among manufacturing in different shipyards, a fuzzy logic approach is used to assess the straightening cost of deck plates. The cost model is implemented and tested in a Java language and is going to be implemented in an industrial environment.

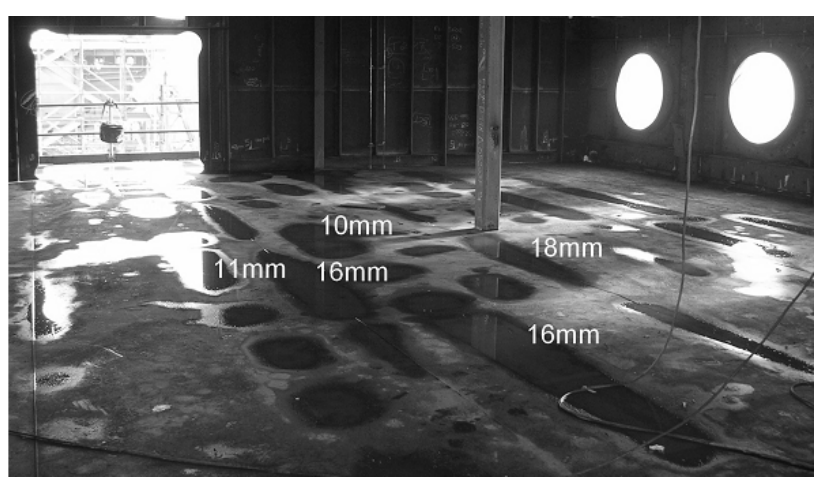

Figure 1 : Distorted ship deck

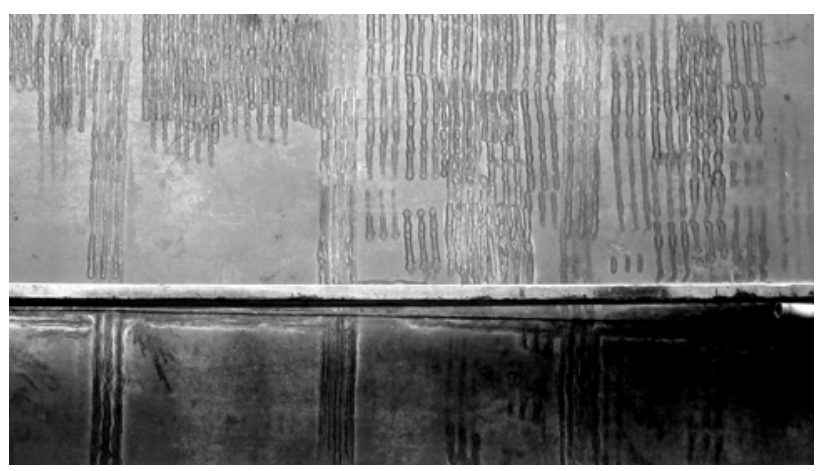

Figure 2 : Straightening heating lines 


\section{CHALLENGES OF PRODUCIBILITY AND COST ASSESSMENT}

The organisation of a production control and cost control system in a shipyard is not an easy task for different reasons [6].

\subsection{UNCOUPLING BETWEEN DESIGN AND COST ENGINEERING}

Cost is often a secondary consideration for the engineers concentrating on delivering the technical aspects of a new design [2]. Indeed, cost evaluation can only be performed once the technical details have been resolved. Moreover, after that, it is possible to review the composition of the design. This two steps process results in a degree of separation between technical and cost engineering departments working on the project and creating the situation where there may be a need for further design iterations. While these two engineering groups (design and cost engineers) operate separately there may be little opportunity to go through an optimisation process to improve the cost.

\subsection{COST EVALUATION IN EARLY DESIGN STAGE}

In most cases the ship construction contracts are signed without the completion of a detailed design. The reason for this is that detailed designs with a detailed cost assessment are very expensive and excessively time consuming [1]. Shipyard work in terms of work specifications is difficult to formalize and predict directly from intricate detailed ship designs.

This induces a very large risk to both the buyer who might end up overpaying a ship and the seller who might have to incur exorbitant costs due to the lack of clear definition of the work.

\subsection{SPECIFICITIES OF THE SHIPBUILDING INDUSTRY}

Cost assessment and production simulation allows management to predict the effectiveness of processes in the shipyard. These methods are most frequently used in industries involved in mass production. This is not the case in shipbuilding, which can be characterized by:

- small series production,

- $\quad$ short time to market,

- many different work disciplines,

- large number of different operations,

- high complexity,

- $\quad$ high degree of manual work,

- difficult working conditions,

- $\quad$ working activities very difficult to identify and quantify.

Thus, because of the production processes more complicated and the production parameters more difficult to quantify, the production simulation is less used in the shipbuilding industry than in certain other industries like automotive industry.

\subsection{COST AND PRODUCIBILITY VARIATION FACTORS}

The construction cost must be tracked during the design process to ensure that the project remains viable to both yard and customer particularly as late changes, introduced into the design, can have considerable cost impacts. The factors on which the cost depends are always changing. Moreover it is only when the production design is finalised that it is possible to make a direct evaluation. The main factors that could result in cost changes are:

- Technology change:

o New production processes

o New materials

o New designs

- Social, economic and political situation:

o Changing workforce (productivity)

o Economic downturn and unrest

- Shipyard backwardness:

o Heavy backwardness causes confusion

o Few orders results in loss of learning

- Labour rates:

o Different for each shipyard

o Effect of learning

o Unpredictable changes

- Material cost:

o Vendor base changes

o High fluctuation of steel rate

- Regulation:

o New rules

- Inflation:

$\begin{array}{ll}\text { o } & \text { Fluctuates unpredictably } \\ \text { o } & \text { Different rate for each item }\end{array}$

The cost assessment in different production assemblies is complicated by the fact that:

- there is insufficient cost data and the quality of this information is often quite low in these early phase

- the data is usually distributed on different ERP and CAM systems which are complicated to handle

- $\quad$ sometimes the required cost information exists only in printed tables, or even in the knowledge of a single expert

- the production process are changing continuously in a shipyard so that the historical cost database cannot be used a long time

- different types of ships induce different types of cost and it is often impossible to compare their relative cost data 
These circumstances cause huge difficulties to assess as accurately as possible the producibility of a ship during the design process.

\section{FUZZY LOGIC FUNDAMENTALS}

\subsection{INTRODUCTION}

Fuzzy logic is an essentially pragmatic, effective and generic approach [5]. It allows systematisation of empirical knowledge which is often hard to control. The theory of fuzzy sets offers a suitable method easy to implement in real time applications and that enables to transcribe the knowledge of designers and operators into dynamic control systems.

While most conventional methods of cost estimation are deterministic in nature, the design process is characterized by intrinsic uncertainties. Fuzzy logic has been used to address the issue of uncertainty in some design applications [9, 11]. In this approach, a mapping between the characteristic parameters of a design and the cost function is achieved through a set of "if-then" rules that incorporates fuzzy logic in terms of varied degrees of membership of the parameters in the cost function.

\subsection{PRINCIPLE AND PROCESS}

The more complex a system, the more difficult it is to make precise assertions on its behaviour. The following are naturally deduced from these observations:

- rather than modelling the system, it is often more useful to model the behaviour/knowledge of a human operator used to control the system;

- rather than using equations, operation can be described by qualitatively with an appropriate quantitative translation.

The theory of fuzzy sets is based on the notion of partial membership: each element belongs partially or gradually to the fuzzy sets that have been defined. The outlines of each fuzzy set (see Figure 3) are not "crisp", but "fuzzy" or "gradual".

A fuzzy set is defined by its membership function. A common example is shown in Figure 5 where we define a set of people of "medium height". In classical logic, we would agree for example that people of medium height are those between $1.60 \mathrm{~m}$ and $1.80 \mathrm{~m}$ tall. The characteristic function of the set (see Figure 4 (a)) gives 0 for heights outside the range $[1.60 \mathrm{~m}, 1.80 \mathrm{~m}]$ and 1 for heights in that range. The fuzzy set of people of "medium height" will be defined by a membership function which differs from a characteristic function in that it can assume any value in the range $[0,1]$. Each possible height will be assigned a degree of membership to the fuzzy set of "medium heights" (see Figure 4 (b)) between 0 and 1.

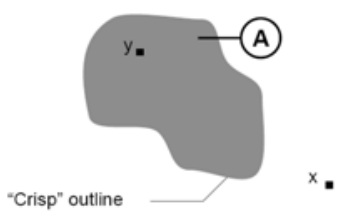

(a) Conventional set

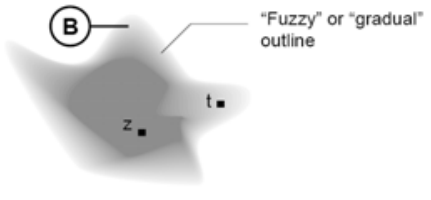

(b) Fuzzy set
Figure 3 : Comparison of a conventional set and a fuzzy set [5]

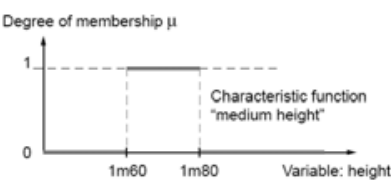

(a) Classical logic (b) Fuzzy logic

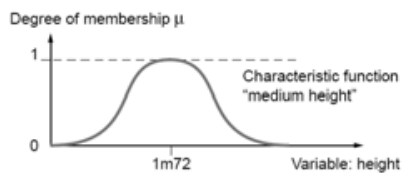

Figure 4 : Fuzzy membership function [5]

A number of fuzzy sets can be defined on the same variable, for example the sets "small height", "medium height" and "tall height", each notion being explained by a membership function (see Figure 5). The variable (for example: height) as well as the terms (small, medium, tall) defined by the membership functions, are known as linguistic variable and linguistic term respectively. Both linguistic variables and terms can be used directly in rules. Fuzzification enables a real value (horizontal axis) to be converted into a fuzzy one (vertical axis).

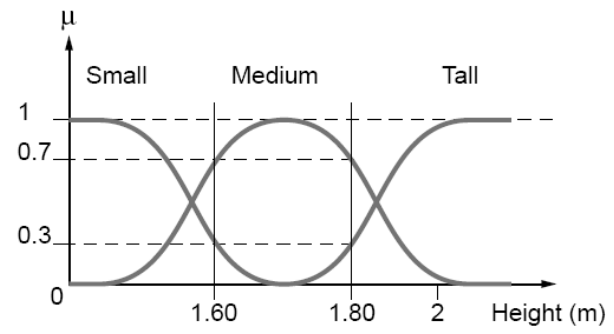

Figure 5 : Membership function, variable and linguistic term [5]

Fuzzy systems provide a non-linear mapping between crisp input variables and crisp output variables and allow the use of linguistic expressions for the rules which define the input-output relationship. A fuzzy system consists of four steps:

- Fuzzification of the crisp input parameters

- Activation of the appropriate fuzzy rules

- The use of the fuzzy inference

- The defuzzification to produce crisp output

In a fuzzy system, fuzzification is the process of transposing crisp input values to true values within relevant fuzzy input sets. Figure 5 shows a simple example with the height of human.

The next step in the fuzzy process is to establish the fuzzy rules. Fuzzy rules are in form of linguistic expressions, which interpret the linguistic input information and 
provide linguistic output information. Fuzzy rules are used in parallel and have the form of "IF predicate THEN conclusion WITH weighting factor". A predicate is a combination of input and output parameters by AND, OR, NOT operators. A rule is activated when the value of the input variable falls totally or partially in a fuzzy set.

The fuzzy rules can be optionally affected by a weighting factor. This factor may vary between $[0,1]$ and states the degree of importance, credibility or confidence of a linguistic rule. The weighting factor shall reduce the membership degree of the conclusion by multiplication of the result with the weighting factor. In order to manipulate the fuzzy control application parameters externally the weighting factor can be a variable. It is therefore possible to modify the output of the fuzzy software just by acting on the weighting factor.

Fuzzy inference is the process that determines the activating level of the consequent output fuzzy set. The degree of activation of a rule is the evaluation of the predicate of each rule by logic combination of the predicate proposals. The "AND" is performed by realising the minimum between the degrees of truth of the proposals. The degree of activation of the rule is used to determine the conclusion of the rule: this operation is called the implication. There are several implication operators, but the most common is the "minimum" operator.

The final step, deffuzyfication, provides the final crisp output. A number of methods can be used, the most common of which is calculation of the "centre of gravity" of the fuzzy set.

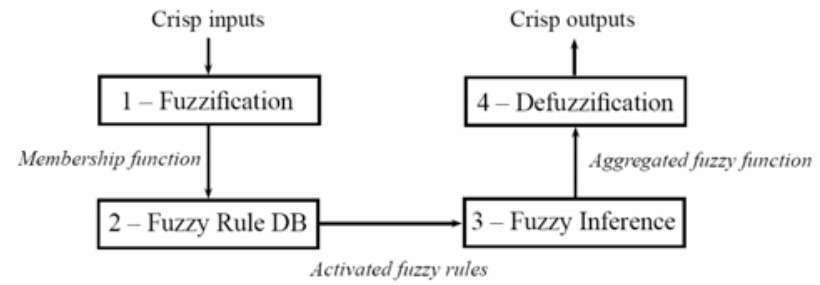

Figure 6 : Schematic diagram of a fuzzy logic system

\subsection{STRONG POINT AND DRAWBACK}

A vital condition for the use of fuzzy rules is the existence of human expertise and know-how. Fuzzy rules bases cannot provide a solution when no-one knows how the system operates or people are unable to manually control it.

When such know-how exists and can be transcribed in the form of fuzzy rules, fuzzy logic simplifies its implementation, and the operation is then easily understood by the user. From the point of view of interpretability it is never perceived as a "black box". This is very good if customers require a detailed list of the reasons and assumptions behind the cost estimate [10].

If human expertise exists, then fuzzy rules can be used, particularly when the system knowledge is tainted by imperfections, when the system is complex and hard to model or when the method used requires a global view of various aspects.

Last but not least, fuzzy logic don't require a large learning database in order to be effective, which would suit industries that produce limited product ranges like shipbuilding industry. Thus, this producibility method can cope easily with novelty or innovation.

\section{STRAIGHTENING FUZZY METRIC}

\subsection{FUZZY SETS AND MEMBERSHIP FUNCTION}

Fuzzy variables are the input and output variables of the fuzzy system. There are two input variables respectively the plate thickness (pt) and the stiffener spacing (ss), and one single output, the relative cost of straightening ( $f_{c}$ ) expressed in man*hour $/ \mathrm{m}^{2}$. The resulting membership functions are defined in Figure 7.
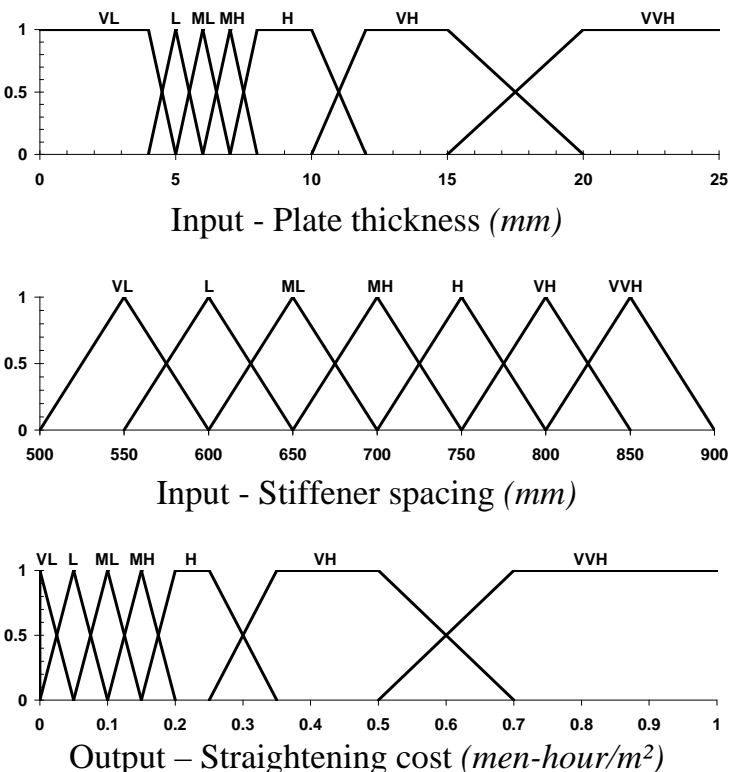

Figure 7 : Fuzzy membership functions of plate thickness, stiffener spacing and relative straightening cost

The input fuzzy sets are defined linguistically as follows:

- $p t$ : [VeryLow (VL), Low (L), MidLow (ML), MidHigh (MH), High $(\mathrm{H})$, VeryHigh $(\mathrm{VH})$, VeryVeryHigh (VVH)]

- ss: [VeryLow (VL), Low (L), MidLow (ML), MidHigh (MH), High (H), VeryHigh (VH), VeryVeryHigh (VVH)] 
The output fuzzy sets are defined linguistically as the relative straightening cost from 0 to $1 \mathrm{man} *$ hour $/ \mathrm{m}^{2}$ :

- $f_{c}$ : [VeryLow (VL), Low (L), MidLow (ML), MidHigh (MH), High (H), VeryHigh (VH), VeryVeryHigh (VVH)]

\subsection{FUZZY RULE MATRIX}

Fuzzy rules are in form of linguistic expressions, which interpret the linguistic input information and provide linguistic output information. The fuzzy rule matrix for the scalar straightening metric has been established in Figure 9 by various experts' opinions of a European shipyard (49 rules). The matrix represents the straightening cost for different combinations of stiffener spacings and plate thicknesses.

\begin{tabular}{|c|c|c|c|c|c|c|c|c|}
\hline & \multicolumn{7}{|c|}{ Stiffener spacing } \\
\hline & & VL & $\mathbf{L}$ & $\overline{M L}$ & $\overline{\mathrm{MH}}$ & $\mathrm{H}$ & VH & $\overline{\text { VVH }}$ \\
\hline \multirow{7}{*}{$\begin{array}{c}\text { Plate } \\
\text { Thickness }\end{array}$} & VL & $\mathrm{VH}$ & $\overline{\mathrm{VH}}$ & $\overline{\mathrm{VH}}$ & $\overline{\mathrm{VH}}$ & $\mathrm{VVH}$ & $\overline{\mathrm{VH}}$ & $\overline{\mathrm{VH}}$ \\
\hline & $\mathbf{L}$ & $\mathrm{H}$ & $\mathrm{H}$ & $\mathrm{H}$ & $\mathrm{H}$ & $\mathrm{VH}$ & $\mathrm{VVH}$ & $\mathrm{VVH}$ \\
\hline & ML & $\mathrm{MH}$ & $\mathrm{H}$ & $\mathrm{MH}$ & $\mathrm{MH}$ & $\mathrm{H}$ & $\mathrm{VH}$ & $\mathrm{VVH}$ \\
\hline & $\mathrm{MH}$ & $\mathrm{ML}$ & $\mathrm{MH}$ & $\mathrm{ML}$ & $\mathrm{ML}$ & $\mathrm{MH}$ & $\mathrm{H}$ & $\mathrm{VH}$ \\
\hline & $\mathrm{H}$ & $\mathrm{L}$ & $\mathrm{ML}$ & $\mathrm{L}$ & $\mathrm{ML}$ & $\mathrm{MH}$ & $\mathrm{MH}$ & $\mathrm{H}$ \\
\hline & VH & $\mathrm{VL}$ & $\mathrm{L}$ & $\mathrm{L}$ & $\mathrm{L}$ & $\mathrm{MH}$ & $\mathrm{MH}$ & $\mathrm{MH}$ \\
\hline & VVH & $\mathrm{VL}$ & $\mathrm{VL}$ & $\mathrm{L}$ & $\mathrm{L}$ & $\mathrm{ML}$ & $\mathrm{ML}$ & $\mathrm{ML}$ \\
\hline
\end{tabular}

Figure 8 : Output fuzzy rule matrix

In this application case we used a Center Of Gravity (COG) defuzzyfication method.

\subsection{OVERALL PERFORMANCE OF FUZZY ESTIMATOR}

The overall performance of the fuzzy straightening metric estimator can be observed by looking at the nonlinear input-output surface in Figure 9. This shows the output cost as a function of the two input variables respectively stiffener spacing and plate thickness. All regions measured between 5 and $25 \mathrm{~mm}$ of plate thickness and between 500 and 900 of stiffener spacing are considered as feasible. The fuzzy system acts to interpolate among the specific cost information obtained for the various stiffened panel configuration (plate thickness, stiffener spacing) using the rule matrix defined by expert opinion.

\section{COMPARISION WITH REAL DATA}

As fuzzy logic required human expertise and know-how in order to define the fuzzy membership functions and the fuzzy linguistic rules, it is common that the output does not fit perfectly with reality. Therefore, we compared the results of fuzzy function with real data.

A database was first of all constituted starting from 1000 measures of straightening cost of 15 passenger ships build in European shipyards. The average straightening cost of 150 points with various combinations between plate ticknesses and stiffener spacings were therefore obtained. Figure 10 shows these points on the output decision surface. Note that the points below the surface are not visible.

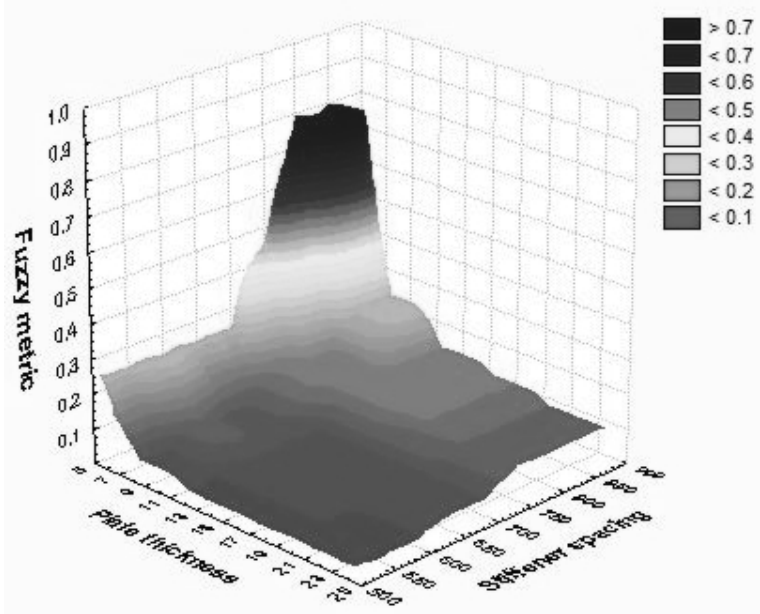

Figure 9 : Decision surface of the straightening metric

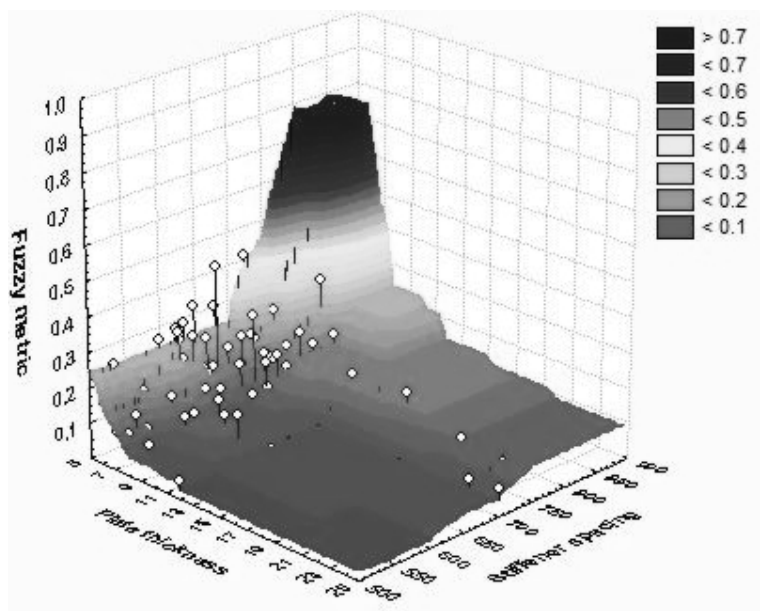

Figure 10 : Decision surface with real cost data

An error function was then defined following the equation (1). This function return an evaluation of the gap between the fuzzy logic output surface and the measured straightening cost (150 points). The result is an error of 1.12 for our application case.

$$
\text { error }=\sqrt{\sum_{i=1}^{n}\left(S_{\text {fuzzy }}-S_{\text {real }}\right)^{2}}
$$

Where:

error Error measured between the output of the fuzzy logic output surface and the measured straightening cost

$n$

Number of points where we know the average cost of straightening

$S_{\text {fuzzy }} \quad$ Average straightening cost evaluated starting from the fuzzy rules

$S_{\text {real }} \quad$ Average straightening cost measured in the shipyard 


\section{6 \\ OPTIMIZATION OF THE FUZZY OUTPUT}

In order to minimize the error of the fuzzy rules in respect with the real measures of straightening costs we have implemented an optimisation module.

The objective function is defined by equation (1) and represents the gap between the fuzzy logic output surface and the measured straightening cost. One design variable was activated in each fuzzy rule which is the weighting factor. An example of a fuzzy rule is shown here: "IF Stiffener spacing IS MidLow AND Plate thickness is High THEN Straightening IS Low WITH 0.456". The weighting factor, varying between $[0,1]$, shall reduce the membership degree of the conclusion by multiplication of the result with the weighting factor. So that he can act on the shape of the fuzzy decision surface.

To avoid deformations of the decision surface where there are no data points coming from measurements, we have automatically added a grid of points with zero error. The algorithm put a new zero error point only if the distance from existing points is upper than $4 \mathrm{~mm}$ along the axis of plate thickness and upper than $50 \mathrm{~mm}$ along the axis of the stiffener spacing. The gap between each point of the grid was chosen as $2 \mathrm{~mm}$ along the axis of plate thickness and $50 \mathrm{~mm}$ along the axis of the stiffener spacing. The combination between the measured point and the grid of zero error point is shown on Figure 12.

A jump optimisation algorithm was used here. It is not a very efficient algorithm but most of the time works better than gradient descent. There is some additional research and development that could be carried out to improve the efficiency of the optimisation algorithm.

The convergence of the objective function is shown in Figure 11. A $26 \%$ reduction of the error has been observed. It means that the decision surface fit better with the measured data than before. Figure 12 shows the optimised output decision surface.

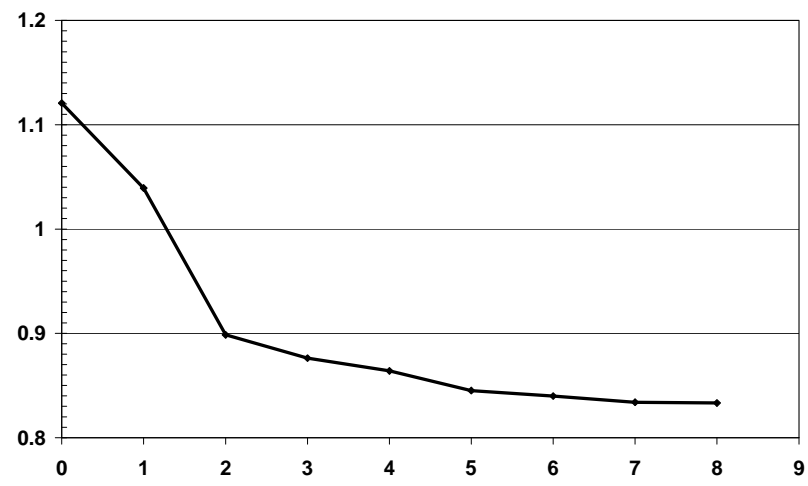

Figure 11 : Convergence of the error objective function

The highest variation of the decision surface has been observed where the concentration of the measuring points is greatest, i.e. for plate thickness between 5 and $11 \mathrm{~mm}$ and for stiffener spacing between 500 and 750 $\mathrm{mm}$.

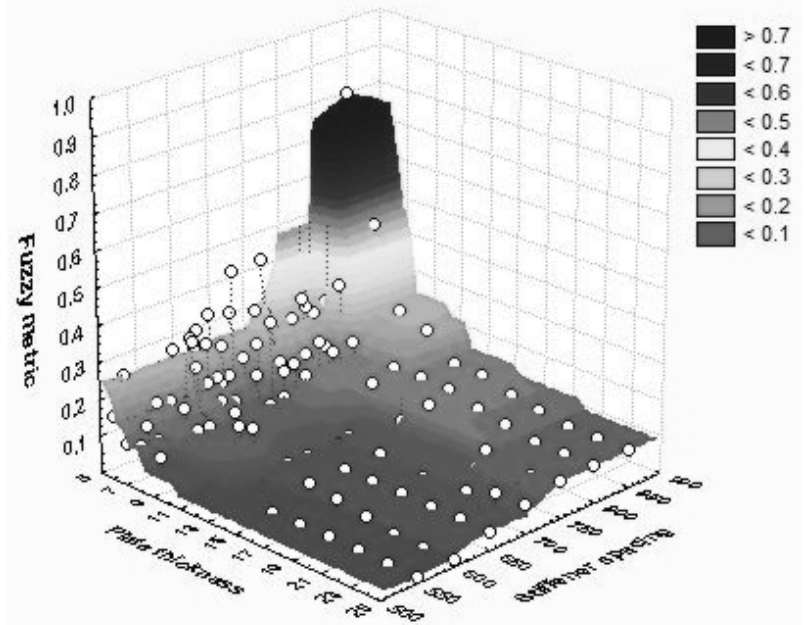

Figure 12 : Optimized decision surface with real cost data

\section{APPLICATION CASE}

To investigate the effect of different types of ships on the fuzzy metric, some specific examples were considered. Amidships sections of three different types of ships, one cruise liner, one Ferry and one LNG have been considered. The three amidships section of 30 meters length are illustrated in Figure 13.

For the computations, the different kind of ships has been subdivided into stiffened panels and pillars. The straightening cost was only computed for the stiffened panels. Figure 13 shows the number of stiffened panel as well as the total weight of the half amidships sections.

When this data is processed through the straightening producibility fuzzy metric system the resulting relative cost per ton can be assessed as shown in Table 1. Two different decision surfaces have been considered, the first one without the optimisation of the weighting factor and the second one with the optimisation of the weighting factor.

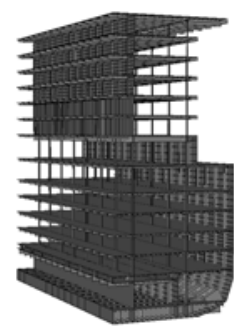

Cruise liner

77 stiffened panels 21 pillars 1458 tons

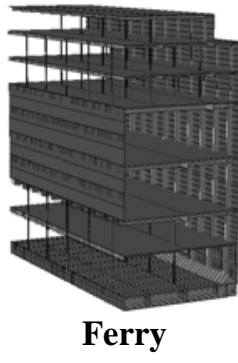

45 stiffened panels 11 pillars 623 tons

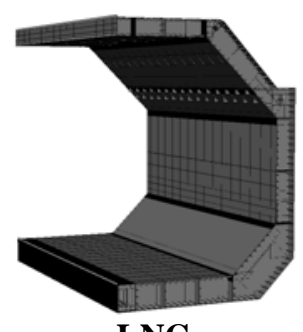

LNG

64 stiffened panels 0 pillars

Figure 13 : Amidships section of three types of ships 
The results indicate that the straightening cost per ton of steel is much greater for the Ferry than for the LNG. The Cruise liner has an intermediate result. The higher cost of straightening is explained by the fact that the minimum plates thickness is $5 \mathrm{~mm}$ for the Ferry while the minimum plate thickness for the cruise liner is $6 \mathrm{~mm}$ and $10 \mathrm{~mm}$ for the LNG.

The difference between the initial fuzzy metric and the optimised fuzzy metric is also highlighted. The results show a difference of $6 \%$ for the cruise liner and $-2 \%$ for the Ferry, while the results for the LNG remain unmodified.

\begin{tabular}{|l|c|c|c|}
\hline & Cruise liner & Ferry & LNG \\
\hline Initial fuzzy metric & 0.90 & 1.26 & 0.32 \\
\hline Optimised fuzzy metric & 0.84 & 1.28 & 0.32 \\
\hline
\end{tabular}

Table 1 : Comparison of the fuzzy straightening metric in hour per tons

If we consider that the actual average cost for steel working (cutting, assembling and welding of steel) in European shipyard is around 50 hour/ton, the estimated straightening cost for the cruise liner, the Ferry and the LNG are respectively of $1.7 \%, 2.6 \%$ and $0.6 \%$.

\section{CONCLUSIONS}

The basic structures used in shipbuilding are stiffened panels that are assembled by welded joints. The welding operations generate distortions that have to be eliminated for esthetical and service reasons. The common use of thin plates increases the straightening operations and it seems thus important to characterise those supplementary works that may induce additional costs and delivery delays.

A fuzzy straightening cost metric has been introduced. This metric can be used to compare the relative costs of different design alternatives for stiffened panels. Because it is defined on a relative cost per unit area basis, it can also be use to compare the relative productivity of different ship scantling. The metric utilizes the scantling of stiffened panel available within current basic design software, but provides a single metric which may be much more useful in the earliest stages of the design process.

The straightening cost information was developed here by using expert opinion based on accumulated experience. For a particular shipyard, specific production cost data could be gathered in order to provide a more accurate basis for the metric. Indeed, we illustrates that it is possible to minimize the errors between the fuzzy output surface and the real measured data trough the modification of the weighting factor of the fuzzy rules.

Since the cost data is naturally imprecise and the actual production process to be used for a specific deck would vary with the equipment, practices, and production schedules choices in each shipyard, a fuzzy approach was utilized to systematically handle this natural imprecision.

Those generated surface response are useful to improve the research in the following domains: production simulation, cost assessment of ship hull, structure optimisation, design for production, etc. Moreover, the advantages of the straightening assessment formula are the rapidity and the simplicity that are very important points in industry. Indeed, it permits to estimate rapidly the value straightening operations.

\section{ACKNOWLEDGEMENTS}

The authors thank University of Liege and experts of some European shipyards for the collaboration in this project as well as the European MARSTRUCT project $\left(n^{\circ}\right.$ TNE3-CT-2003-506141) and the European IMPROVE project (nº31382- FP6 2005 Transport-4).

\section{REFERENCES}

1. BARENTINE, J., A Process-Based Cost Estimating Tool for Ship Structural Designs, Master's thesis, Massachusetts Institute of Technology (MIT), 1996

2. BOLE, M., Cost Assessment at Concept Stage Design Using Prametrically Generated Production Product Models, ICCAS07, 2007

3. BRUCE, G.J., YULIADI, M.Z., and SHAHAB, A., Towards a Practical Means of Predicting Weld Distortion, Journal of Ship Production, Vol. 17, No. 2, May 2001, pp. 62-68

4. CHAU T.T., JANCART F. and BECHEPAY G., About the welding effects on thin stiffened panel assemblies in shipbuilding. In: International Conference on Marine Technology, 2001, Szczecin, Poland.

5. CHEVRIE, F. and GUELY, F. (1998). Fuzzy Logic. Schneider-Electric, 1998

6. FERGUSON, W. B., Shipbuilding Cost \& Production Methods, Cornell Maritime Press, 1944

7. KMIECIK, M., JASTRZBSKI, T., AND KUNIAR, J., Statistics of Ship Plating Distortions, Marine Structures, vol 8 (1995) 119-132

8. LOSSEAU, N. and CAPRACE, J.D., A data mining analysis to evaluate the additional workloads caused by welding distortions. In: MARSTRUCT Conference, 2009, Lisbon, Portugal

9. PARSONS, M. G., NAM, J.-H., and SINGER, D. J., A Scalar Metric for Assessing the Productivity of a Hull Form in Early Design, Journal of ship production, 1999, 15(2):91-102. 
10. ROY, R., and KERR, C., Cost Engineering: Why, What and how?, 2003,Cranfiel University.

11. SHEHAB, E. and ABDALLA, H., An Intelligent Knowledge-Based System for Product Cost Modelling, The international journal of advanced manufacturing technology, 2002.

\section{AUTHORS BIOGRAPHY}

Jean-David Caprace holds the current position of research engineer at ANAST department of University of Liege (ULg). He is responsible for production simulation and design for production researches. His previous experience includes the involvement in various European research project like InterSHIP, IMPROVE, MARSTRUCT and he is currently a member of the ISSC committee V.3 - "Materials and fabrication technology".

Fransisco Aracil Fernandez recently graduated holds now the current position of research engineer at ANAST department of University of Liege (ULg). During his studies he was involved in European project like MARSTRUCT and VISION.

Nicolas Losseau holds the current position of research engineer at ANAST department of University of Liege (ULg). He is responsible of the production simulation and the analyses of welding distortion. His previous experience includes European project InterSHIP, IMPROVE, and MARSTRUCT.

Philippe Rigo is a full professor at University of Liege (ULg), Belgium, ANAST- Naval Architecture Research Unit. He coordinates various projects as the IMPROVE EU-FP6 project and leads the research unit in ship structure analysis and more specifically in ship structure least cost optimisation (LBR5 Software). He is member of ISSC since 1991, mainly in Ultimate Strength Committees. 